\title{
ACCIÓN HOLOMORFA AFÍN QUE PROVIENE DE UNA ACCIÓN DIFERENCIAL AFÍN SOBRE $\mathrm{C}^{m}$
}

\section{HOLOMORPHIC AFFINE ACTION THAT COMES FROM A DIFFERENTIAL ACTION AFFINE ON $\mathrm{C}^{m}$}

\author{
Alessandri Canchoa Q. ${ }^{1}$ \& Benito L. Ostos C. ${ }^{2}$
}

\section{Resumen}

Dada una acción diferencial afín $\varphi: A f f(\mathrm{R})^{+} \times \mathrm{C}^{m} \rightarrow \mathrm{C}^{m}$, tal que para todo $(s, t) \in \operatorname{Aff}(\mathrm{R})^{+}$, $\varphi_{(s, t)}(z)$ pertenece al grupo de automorfismos de $\mathbb{C}^{m}$. Se sabe que esta acción asocia dos campos diferenciables $X$ e $Y, \mathrm{R}$-completos que están relacionados por el corchete de Lie mediante la relación $[X, Y]=-Y$. Usando la estructura casi-compleja de $\mathbb{C}^{m}$, se definen los campos $J X, J Y$ y luego los campos $\tilde{X}=$ $\frac{1}{2}(\mathrm{X}-i J \mathrm{X})$ e $\tilde{Y}=\frac{1}{2}(\mathrm{Y}-i J \mathrm{Y})$ en $\mathbb{C}^{m}$.

Se resuelve el siguiente problema: Si $[J X, J Y]=Y$ con $\tilde{X}$ cuyo flujo es periódico de período $2 \pi \mathrm{i}$, entonces $\tilde{\varphi}=\operatorname{Aff}(\mathrm{C}) \times \mathrm{C}^{m} \rightarrow \mathrm{C}^{m}$ dado por $\tilde{\varphi}_{(s, t)}=\mathrm{Y}_{t}{ }^{\circ} X_{l n s}$, es una acción holomorfa afín.

Palabras claves: Acción diferencial afín, campos vectoriales $R$-completos, corchete de Lie.

\section{Abstract}

Given an affine differential action $\varphi: A f f(\mathrm{R})^{+} \times \mathrm{C}^{m} \rightarrow \mathrm{C}^{m}$, such that for all $(s, t) \in \operatorname{Aff}(\mathrm{R})^{+}$, $\varphi_{(s, t)}(z)$ belongs to the group of automorphisms of $\mathbb{C}^{m}$. It is known that this action associated two differentiable fields $X$ and $Y, \mathrm{R}$-complete than are related by the Lie bracket by the relation $[X, Y]=-Y$. Using the quasi-complex structure of $\mathbb{C}^{m}$, fields $J X, J Y$ are defined, then the fields campos $\tilde{X}=\frac{1}{2}(\mathrm{X}-i J \mathrm{X})$ and $\tilde{Y}=\frac{1}{2}(\mathrm{Y}-i J \mathrm{Y})$ are defined in $\mathbb{C}^{m}$.

Solves the following problem: If $[J X, J Y]=Y$ with $\tilde{X}$ whose flow is periodic of period $2 \pi \mathrm{i}$, then $\tilde{\varphi}=$ $\operatorname{Aff}(\mathrm{C}) \times \mathrm{C}^{m} \rightarrow \mathrm{C}^{m}$ given by $\tilde{\varphi}_{(s, t)}=\mathrm{Y}_{t}{ }^{\circ} X_{l n s}$, is a holomorphic affine action.

Keywords: Action differential affine, $\mathrm{R}$-complete vector fields, Lie bracket.

\section{Introducción}

Este artículo está dedicado a encontrar condiciones necesarias para que una acción diferencial afín sobre una variedad compleja induzca una acción holomorfa afín. La importancia de este trabajo radica en el hecho que se pueda conectar campos diferenciables con campos holomorfos y usar técnicas para resolver problemas de uno o del otro lado.

Una acción diferencial afín es una acción diferencial $\varphi: \operatorname{Aff}(\mathrm{R})^{+} \times M \rightarrow M$ del grupo $\operatorname{Aff}(\mathrm{R})^{+}$ sobre una variedad diferenciable $M$. Se sabe que esta acción asocia dos campos $\mathrm{R}$-completos y que

\footnotetext{
${ }^{1}$ Universidad Nacional Agraria La Molina, Lima, Perú. E-mail:canchoa@lamolina.edu.pe

${ }^{2}$ Universidad Nacional Agraria La Molina, Liam, Perú. E-mail: benitoostos@1amolina.edu.pe
} 
están relacionados por el corchete de Lie mediante la relación $[X, Y]=-Y$. Esto implica que podemos definir u a foliación diferencial de codimensión $\operatorname{dim} M-2$. Cuando $M$ es una variedad compleja, se puede usar la estructura casi-compleja de $M, J$, para definir campos $J X$ y $J Y$ que no necesariamente son $\mathrm{R}$-completos. Cuando $\varphi_{(s, t)}$ pertenece al grupo de automorfismos de $M$ (denotado por $\operatorname{Aut}(M)$ ), los campos $X$ y $J X$ son conmutativos (lo mismo para $Y$ y $J Y$ ). Los campos $\tilde{X}=\frac{1}{2}(\mathrm{X}-i J \mathrm{X})$ e $\tilde{Y}=\frac{1}{2}(\mathrm{Y}-i J \mathrm{Y})$ son holomorfos pero no son necesariamente $C$ -completos. Se puede definir una aplicación multivaluada $\tilde{\varphi}=\operatorname{Aff}(\mathrm{C}) \times M \rightarrow M$, dado por $\tilde{\varphi}_{(s, t)}=\tilde{Y}_{t}^{\circ} \tilde{X}_{l n s}$, para todo $\left.\mathbf{9}, t\right)$ Affe( contribución en este trabajo es el siguiente resultado:

Sea $\varphi=A f f(\mathrm{R})^{+} \times \mathrm{C}^{m} \rightarrow \mathrm{C}^{m}$ una acción diferencial afín tal que $\varphi_{(s, t)} \in A u t\left(\mathrm{C}^{m}\right)$, para todo $(s, t) \in A f f(\mathrm{R})^{+} . \mathrm{Si}[J X, J Y]=Y$ con $\tilde{X}$ cuyo flujo es $2 \pi \mathrm{i}$ periódico, entonces $\tilde{\varphi} e \mathrm{~s}$ una acción holomorfa afín.

\section{Materiales y métodos \\ Preliminares}

En esta sección se enuncian las definiciones y propiedades que se usarán a lo largo del trabajo.

\section{Campos vectoriales diferenciables en $R^{m}$.}

Para cada $x=\left(x_{1}, x_{2}, \cdots, x_{m}\right)$ en $\mathrm{R}^{m}$,

consideremos la base canónica $\left\{\frac{\partial}{\partial x_{1}}, \cdots, \frac{\partial}{\partial x_{m}}\right\}$ del

espacio tangente $T_{x} \mathrm{R}^{m}$, que por definición es el

espacio vectorial $\{x\} \times \mathrm{R}^{m}$.

Definición 1 Un campo vectorial diferenciable $X$ en un abierto $U$ de $\mathrm{R}^{m}$, es una aplicación diferenciable que a cada punto $x$ le asocia un vector tangente $X(\mathrm{x})$ de $T_{x} \mathrm{R}^{m}$.

Es decir $X$ es:

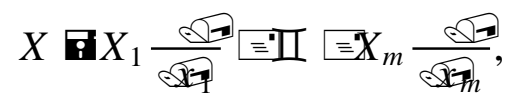

donde $X_{1}, \cdots, X_{m}$ son funciones diferenciables en $U$.

Asociado a $X$ tenemos el siguiente sistema de ecuaciones diferenciales

$$
\frac{d x}{d t}=X(x)
$$

Teorema 1 (Teorema de existencia y unicidad)

Para el sistema anterior se verifican:

1. Sea $U \subset \mathbb{R}^{m}$ un conjunto abierto. Para cualquier $x \in U$ existe un intervalo abierto $I_{x}=\left(a_{x}, b_{x}\right) \subset$ $\mathbb{R}$, y una función diferenciable $\varphi_{x}: I_{x} \rightarrow U$ tal que $\varphi_{x}(0)=x \quad, \quad$ y $\quad \frac{d}{d t} \varphi_{x}(t)=X\left(\varphi_{x}(t)\right)$, para cada t en $I_{x}$.

2. Si existe otra solución local $\varphi_{x}^{\prime}: I_{x}^{\prime} \rightarrow U$ de $X$ en $x$, entonces $\varphi_{x}$ y $\varphi_{x}^{\prime}$ coinciden en $I_{x} \cap I_{x}^{\prime}$.

Demostración.-. Veáse en [7, pp. 17]

La función $\varphi_{x}: I_{x} \rightarrow U$ es llamada solución local de (1) que pasa por $x$.

A partir de este teorema se verifica lo siguiente: para cada $x \in U$ existe una única solución $\varphi_{x}: I_{x} \rightarrow U$ con la propiedad de que cualquier otra solución $\psi: I \rightarrow U$ de (1) satisface $I \subset I_{x}$ y $\psi=\left.\varphi_{x}\right|_{I}$. La solución $\varphi_{x}$ es llamada solución máximal de la ecuación (1) que pasa por el punto $x$. En este caso el intervalo máximal se denotará por $I_{x}=(\alpha(x), \omega(x)) \quad$. $\quad$ Las funciones $-\alpha(x), \omega(x): U \rightarrow(0,+\infty) \quad$ son semicontinuas inferiores. Si $\omega(x)<\infty$ para algún $x \in U$ entonces $\varphi_{x}(t)$ escapa de cualquier conjunto compacto en $U$ cuando $t$ tiende a $\omega(x)$. Similarmente, si $\alpha(x)>-\infty$ para algún $x \in U$ entonces $\varphi_{x}(t)$ escapa de cualquier conjunto compacto en $U$ cuando $t$ tiende a $\alpha(x)$. El conjunto $\Omega=\{(t, x) \in \mathrm{R} \times U: \alpha(x)<t<\omega(x)\}$ es llamado dominio fundamental del campo $X$. En este conjunto se define la aplicación diferenciable:

$$
\begin{array}{clc}
\varphi: \Omega \subset \mathrm{R} \times U & \rightarrow & U \\
(t, x) & \mapsto & \varphi(t, x):=\varphi_{x}(t)
\end{array}
$$

y satisface lo siguiente:

- $\varphi(0, x)=x$ para todo $x$ en $U, \mathrm{y}$

- $\varphi(t, \varphi(s, x))=\varphi(t+s, x)$, donde quiera que ambos miembros estén definidos. A $\varphi$ se le denomina el flujo del campo $X$. En adelante se denotará $X_{t}(p)=\varphi(t, p)$.

Definición 2 Se dice que $X$ es un campo $\mathrm{R}$ completo si en la definición anterior $\Omega=\mathrm{R} \times U$. 
Definición 3 Sean $M$ y $N$ variedades diferenciales y $X, Y$ dos campos vectoriales sobre $M$ y $N$ respectivamente. Si $p$ y $q$ son dos puntos de $M$ y $N$ respectivamente, se dice que $X$ e $Y$ son localmente conjugados en $p$ y $q$ si existen dos conjuntos abiertos $U \subset, \quad V \subset N \quad, \quad p \in U, \quad q \in V \quad$ y $\quad$ un difeomorfismo $\Phi: U \rightarrow V, \quad \Phi(p)=q$ tal que $D \Phi \circ\left(\left.X\right|_{U}\right)=\left.Y\right|_{V} \circ \Phi$.

Teorema 2 (Teorema del flujo tubular) Sea $X$ un campo vectorial diferencial sobre la variedad $M$ y un punto $p \in M$ tal que $X(p) \neq 0$. Entonces $X$ es localmente conjugada en $p$ y $0 \in \mathbb{R}^{m}$ al campo constante $\frac{\partial}{\partial x_{1}}$.

Para la prueba véase [7].

Definición 4 Sean $M$ y $N$ variedades diferenciales y $f: M \rightarrow N$ un difeomorfismo. Si $X$ es un campo vectorial de clase $C^{\infty}$ en $M$, se define un campo vectorial $f^{*}(X)$ en $N$ de la misma clase dado por $f^{*}(X)(p)=D f^{-1}(f(p)) \cdot X(f(p))$, para todo $p \in N$.

Definición 5 Sea $X$ e $Y$ dos campos en $M$. Fijando un punto $p \in M$ y otro punto $t \in \mathrm{R}$, el vector

$$
v(t)=X_{t}^{*}(Y)(p)=D X_{-t}\left(X_{t}(p)\right) \cdot Y\left(X_{t}(p)\right),
$$

es tangente a $M$ en $p$.

Definición 6 El corchete de Lie de $X$ e $Y$ es un campo vectorial $[X, Y]$ definido por

$[X, Y](p)=\left.\frac{d}{d t}\left(X_{t}^{*}(Y)(p)\right)\right|_{t=0}$, para todo $p \in M$.

Lema 1 Sea $\mathrm{x}: U \subset M \rightarrow \mathbb{R}^{m}$ una carta local. En esta carta local se puede expresar $X=\sum_{i=1}^{m} a_{i} \frac{\partial}{\partial x_{i}}$ e $Y=\sum_{i=1}^{m} b_{i} \frac{\partial}{\partial x_{i}}$. Entonces $[X, Y]=\sum_{i=1}^{m} c_{i} \frac{\partial}{\partial x_{i}}$, donde

$$
c_{i}
$$

Para la prueba véase [1].

Proposición 1 El corchete de Lie tiene las siguientes propiedades

1. $[$,$] es bilineal: \left[a X_{1}+b X_{2}, Y\right]=a\left[X_{1}, Y\right]+$ $b\left[X_{2}, Y\right] \mathrm{y}$

$\left[X, a Y_{1}+b Y_{2}\right]=a\left[X, Y_{1}\right]+b\left[X, Y_{2}\right]$ donde $a$ y $b$ son constantes.

2. $[X, Y]=-[Y, X]$.

Para la prueba véase [1].

Las definiciones y resultados precedentes se pueden extender si se cambia $\mathrm{R}^{m}$ por $\mathrm{C}^{m}$ y campos diferenciables por campos holomorfos.
Campos vectoriales diferenciables en una variedad compleja

Sea $X$ un campo vectorial diferenciable en una variedad compleja $M$ de dimensión $m$. Sea $T M \oplus \mathrm{C}=T^{(1,0)} M \oplus T^{(0,1)} M$ la descomposición usual del complexificado del fibrado tangente a $M$. Denotemos por $J: T M \rightarrow T M$ el operador de estructura casi-compleja inducida por la estructura compleja sobre $M$. Entonces se tiene un isomorfismo $T M \approx T^{(1,0)} M$ dado por $\mathrm{X} \mapsto \tilde{X}=\frac{1}{2}(\mathrm{X}-i J \mathrm{X})$.

Definición 7 Diremos que un campo vectorial real $X$ sobre $M$ es holomorfo si $\tilde{X}$ es holomorfo.

Si $X$ es holomorfo, se considera la ecuación diferencial asociada a (1):

$$
\frac{d z}{d \zeta}=\tilde{X}(z), z(0)=z^{0} .
$$

La ecuación anterior es equivalente al sistema de ecuaciones diferenciales con condiciones iniciales

$\frac{d z}{d t}=X(z), \frac{d z}{d s}=J X(z), \zeta=t+i s, z(0)=z^{0}$.

donde $\frac{\partial}{\partial \zeta}=\frac{1}{2}\left(\frac{\partial}{\partial t}-i \frac{\partial}{\partial s}\right)$.

Se verifica

$[X, J X]=[\tilde{X}+\tilde{\tilde{X}}, i \tilde{X}-i \overline{\tilde{X}}]=-2 i[\tilde{X}, \overline{\tilde{X}}]=0$,

esto es equivalente a

$$
X_{\hat{t}} \circ J X_{\hat{s}}=J X_{\hat{s}} \circ X_{\hat{t}} \forall(\hat{t}, \hat{s}) \in \mathrm{R} \times \mathrm{R} .
$$

Por consiguiente los flujos de los campos $X$ y $J X$ son conmutativos.

Definición 8 Un campo vectorial real $X$ es $\mathbb{C}$ -completo si $\tilde{X}$ es $\mathbb{C}$-completo.

Si $\tilde{X}$ es $\mathbb{C}$-completo, el flujo de $\tilde{X}, \tilde{X}_{t+i s}(z)=$ $X_{t}{ }^{\circ} Y_{s}(z)=Y_{s}{ }^{\circ} X_{t}(z)$ existe para todo $z \in M \quad \mathrm{y}$ para todo $t+i s \in \mathbb{C}$.

Un campo vectorial $\tilde{X}$ es $\mathbb{C}$-completo si y sólo si $X$ y $J X$ son $\mathbb{R}$-completos.

\section{Acciones afines reales sobre una variedad diferenciable}

Denotemos por $A f f(\mathrm{R})$, el grupo de Lie de transformaciones afines con la composición de funciones afines. Un elemento $f \in A f f(\mathrm{R})$ es definido por $f(x)=s x+t$, donde $s, t \in \mathrm{R} \quad \mathrm{y}$ $s \neq 0$. Dados $f, g \in A f f(\mathrm{R}), f(x)=s_{1} x+t_{1} \quad \mathrm{y}$ $g(x)=s_{2} x+t_{2} \quad, \quad$ la composición $f \circ g(x)=s_{1} s_{2} x+s_{1} t_{2}+t_{1}$ es también afín. Algunas propiedades de este grupo son:

1. Es un grupo de Lie de dimensión dos, con dos componentes conexas, denotados por $\operatorname{Aff}(\mathrm{R})^{+}$, 


$$
\begin{aligned}
& \operatorname{Aff}(\mathrm{R})^{-} \text {y definidos por } \\
& \begin{aligned}
A f f(\mathrm{R})^{+} & =\{f(x)=s x+b: s>0\} \\
\operatorname{Aff}(\mathrm{R})^{-} & =\{f(x)=s x+b: s<0\} .
\end{aligned}
\end{aligned}
$$

2. $A f f(\mathrm{R})^{+}$no es abeliano, esto implica que no es isomorfo a $R^{2}$.

3. $\operatorname{Aff}(\mathrm{R})^{+}$es difeomorfo a $\mathrm{R}^{2}$, esto implica que es simplemente conexa.

Sea el grupo $\left(R_{>0} \times R, \circ\right)$, donde la operación $\circ$ está dado por:

$$
\begin{gathered}
\left(s_{1}, t_{1}\right) \circ\left(s_{2}, t_{2}\right)=\left(s_{1} s_{2}, s_{1} t_{2}+t_{1}\right), \text { para todo } \\
\left(s_{1}, t_{1}\right),\left(s_{2}, t_{2}\right) \in \mathrm{R}_{>0} \times \mathrm{R} .
\end{gathered}
$$

El elemento neutro es $(1,0)$ y el inverso de $(s, t)$ es $(s, t)^{-1}=\left(\frac{1}{s},-\frac{t}{s}\right)$. Además es un grupo no abeliano $\mathrm{y}$ es isomorfo a $\operatorname{Aff}(\mathrm{R})^{+}$.

Definición 9 Sea $G$ un grupo de Lie diferenciable con una operación $*$ y $M$ una variedad diferenciable (o una variedad compleja). Se dice que $\varphi: G \times M \rightarrow M$ es una acción diferenciable del grupo $G$ sobre la variedad $M$ cuando $\varphi$ es un mapeo diferenciable y satisface las siguientes condiciones:

i) $\varphi(e, p)=p$ para todo $p \in M$, donde $e$ es la identidad de $G$.

ii) $\varphi\left(\mathrm{g}_{1} * \mathrm{~g}_{2}, \mathrm{p}\right)=\varphi\left(\mathrm{g}_{1}, \varphi\left(\mathrm{g}_{2}, \mathrm{p}\right)\right)$, para todo $g_{1}, g_{2} \in G$ y para todo $p \in M$.

Definición 10 Una acción diferenciable $\varphi:\left(\mathrm{R}_{>0} \times \mathrm{R}\right) \times M \rightarrow M$, del grupo $\mathrm{R}_{>0} \times \mathrm{R}$ sobre una variedad diferenciable (o una variedad compleja) $M$ de dimensión por lo menos tres es llamado acción diferencial afín.

\section{METODOLOGÍA}

Caracterización de acciones afines reales sobre una variedad diferenciable.

En las dos proposiciones que siguen se da condiciones necesarias y suficientes para que una acción de clase diferenciable, $\varphi:\left(\mathbb{R}_{>0} \times \mathbb{R}\right) \times M \rightarrow M$, sea una acción diferencial afín.

Proposición 2 Sea $\varphi:\left(\mathbb{R}_{>0} \times \mathbb{R}\right) \times M \rightarrow M$ una acción diferenciable afín. Entonces existen campos $\mathbb{R}$-completos $X$ e $Y$ tales que:

1. $[X, Y]=-[Y, X]$.

2. Si $X_{s}$ e $Y_{t}$ denotan los flujos de los campos $X$ e $Y$ entonces $\varphi_{(s, t)}=\mathrm{Y}_{t}{ }^{\circ} X_{l n s}$.
Demostración: Como $(s, t)=(1, t) \circ(s, 0)$, y siendo $\varphi$ una acción se cumple $\varphi_{(s, t)}=\varphi_{(1, t)(s, 0)}=\varphi_{(1, t)} \circ \varphi_{(s, 0)}$.

Luego se puede asociar dos acciones, una en $R_{>0}$ y la otra en $\mathrm{R}$ sobre $M$ :

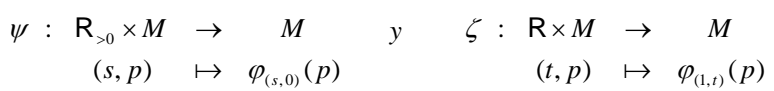

Luego se tiene dos campos $\mathbb{R}$-completos:

$X(z)=\left.\frac{\partial}{\partial s} \varphi_{\left(e^{s}, 0\right)}(z)\right|_{s=0}$ e $Y(z)=\left.\frac{\partial}{\partial t} \varphi_{(1, t)}(z)\right|_{t=0}$,

que satisfacen la relación $[X, Y]=-Y$. En efecto:

$X_{s}^{*}(Y)(p)=\left.\frac{d}{d t}\left(X_{-s} \circ Y_{t} \circ X_{s}\right)(p)\right|_{t=0}=\left.\frac{d}{d t}\left(\varphi_{\left(e^{-s}, 0\right)} \circ \varphi_{(1, t)} \circ \varphi_{\left(e^{s}, 0\right)}\right)(p)\right|_{t=0}$

$$
=\left.\frac{d}{d t} \varphi_{\left(1, e^{-s} t\right)}(p)\right|_{t=0}=\left.e^{-s} \frac{d}{d t} \varphi_{(1, t)}(p)\right|_{t=0}=e^{-s} Y(p) .
$$

Luego

$[X, Y](p)=\left.\frac{d}{d s} X_{s}^{*}(Y)(p)\right|_{s=0}=-\left.e^{-s} Y(p)\right|_{s=0}=-Y(p)$.

De otro lado

$$
\begin{aligned}
\left(Y_{t} \circ X_{\ln s}\right)(p) & =\left(\varphi_{(1, t)} \circ \varphi_{\left(e^{\ln s}, 0\right)}\right)(p) \\
& =\left(\varphi_{(1, t)} \circ \varphi_{(s, 0)}\right)(p) \\
& =\varphi_{(s, t)}(p) .
\end{aligned}
$$

Lema 2 Si $X$ e $Y$ son dos campos $\mathbb{R}$-completos en una variedad $M$ y $[X, Y]=-[Y, X]$ entonces sus flujos están relacionados por: $Y_{e^{s} t} \circ X_{s}=X_{s} \circ Y_{t}$, para todo $\mathrm{s}, \mathrm{t} \in \mathbb{R}$.

Demostración: Por la regla de la cadena se obtiene

$$
\begin{aligned}
\frac{d}{d s} X_{s}^{*}(Y)(p) & =\left.\frac{d}{d t} X_{s+t}^{*}(Y)(p)\right|_{t=0}=\left.\frac{d}{d t} X_{t}^{*} \circ\left(X_{s}^{*} \circ(Y)\right)(p)\right|_{t=0} \\
& =\left[X, X_{s}^{*}(Y)\right](p)=\left[X_{s}^{*}(X), X_{s}^{*}(Y)\right](p) \\
& =X_{s}^{*}([X, Y])(p)=-X_{s}^{*}(Y)(p)
\end{aligned}
$$

entonces $X_{s}^{*}(Y)(p)=e^{-s} Y(p)$.

Por otro lado el flujo de $X_{s}^{*}(Y)$ es $t \rightarrow X_{s}^{-1} \circ Y_{t} \circ X_{s}$ mientras que el flujo de $e^{-s} Y$ es $t \rightarrow Y_{e^{-s} t}$. Luego 
$X_{s^{-1}} \circ Y_{t} \circ X_{s}(p)=Y_{e^{-s} t}(p)$, para todo $s, t \in \mathrm{R}$ y $p \in M$.

Proposición 3 Sean $X$ e $Y$ dos campos $\mathbb{R}$-completo en una variedad $M$ con $[X, Y]=-[Y, X]$. Sea $\varphi:\left(\mathbb{R}_{>0} \times \mathbb{R}\right) \times M \rightarrow M$ una aplicación tal que $\varphi_{(s, t)}=\mathrm{Y}_{t}^{\circ} X_{\text {lns }}$, entonces $\varphi$ es una acción diferencial afín.

La aplicación $\varphi$ es diferenciable ya que los flujos de los campos de $X$ e $Y$ son diferenciables. Sólo falta probar que $\varphi$ es una acción. Sea $p \in M$, se obtiene $\varphi_{(1,0)}(p)=Y_{0} \circ X_{\ln 1}(p)=Y_{0}\left(X_{0}(p)\right)=Y_{0}(p)=p$ y además

$$
\begin{aligned}
\varphi_{\left(s_{1}, t_{1}\right) \circ\left(s_{2}, t_{2}\right)}(p) & =\varphi_{\left(s_{1} s_{2}, s_{1} t_{2}+t_{1}\right)}(p)=Y_{s_{1} t_{2}+t_{1}} \circ X_{\ln \left(s_{1} s_{2}\right)}(p) \\
& =Y_{t_{1}} \circ Y_{s_{1} t_{2}} \circ X_{\ln s_{1}} \circ X_{\ln s_{2}}(p) \\
& =Y_{t_{1}} \circ\left(Y_{e^{\operatorname{lns} t_{2}}} \circ X_{\ln s_{1}}\right) \circ X_{\ln s_{2}}(p)
\end{aligned}
$$

Por el Lema 2, se obtiene

$$
\begin{aligned}
\varphi_{\left(s_{1}, t_{1}\right) \circ\left(s_{2}, t_{2}\right)}(p) & =Y_{t_{1}} \circ X_{\ln s_{1}} \circ Y_{t_{2}} \circ X_{\ln s_{2}}(p) \\
& =\varphi_{\left(s_{1}, t_{1}\right)}\left(Y_{t_{2}} \circ X_{\ln s_{2}}(p)\right) \\
& =\varphi_{\left(s_{1}, t_{1}\right)}\left(\varphi_{\left(s_{2}, t_{2}\right)}(p)\right) \\
& =\left(\varphi_{\left(s_{1}, t_{1}\right)} \circ \varphi_{\left(s_{2}, t_{2}\right)}\right)(p) .
\end{aligned}
$$

El grupo de isotropía de $\varphi$ es definido y denotado por

$$
G_{p}(\varphi)=\left\{(s, t) \in \mathrm{R}_{>0} \times \mathrm{R}: \varphi_{(s, t)}(p)=p\right\} .
$$

Este es un subgrupo cerrado del grupo $\left(\mathrm{R}_{>0} \times \mathrm{R}, \circ\right)$. Además si $G_{p}(\varphi)=\mathrm{R}_{>0} \times \mathrm{R}$ entonces las orbitas de $\varphi$ en cada punto $p \in M$ es el mismo punto $p$ , es decir esta dado por $\mathcal{O}_{p}(\varphi)=p$. En este caso se dice que la acción afín es trivial. Se trabaja sobre acciones no triviales.

Definición 11 La acción $\varphi$ es localmente libre si los grupos de isotropía $G_{p}(\varphi)$ son discretos. La acción es foliada si todas las orbitas $\mathcal{O}_{p}(\varphi)$ tienen la misma dimensión.

$\mathrm{Si} \mathcal{C}, t \ell$ es localmente libre, se sabe que una órbita de $e$

$$
\mathcal{O}_{p}(\varphi)=\left\{\varphi_{(s, t)}(p):(s, t) \in \mathrm{R}_{>0} \times \mathrm{R}\right\}
$$

es una variedad real de dimensión dos. Es difeomorfo a un plano o a un cilindro, es decir, el grupo de isotropía
$G_{p}(\varphi)$ es isomorfo a $\{(1,0)\}$ o a $\{1\} \times \mathbf{Z}$.

Ejemplo $1 \mathrm{Si}$ en el Lema anterior $X$ es el campo radial,

$$
X(x)=x_{1} \frac{\partial}{\partial x_{1}}+x_{2} \frac{\partial}{\partial x_{2}}+x_{3} \frac{\partial}{\partial x_{3}},
$$

entonces el flujo del campo $Y$ cumple la siguiente igualdad:

$$
\begin{gathered}
Y_{t}(x)=e^{-s} Y_{e^{s} t}\left(e^{s} x\right), \text { para todo } \\
(s, t) \in \mathrm{R} \times \mathrm{R}, \quad x=\left(x_{1}, x_{2}, x_{3}\right) \in M .
\end{gathered}
$$

Como las órbitas del campo $X$ son rectas que pasan por el origen de coordenadas, las órbitas de la acción afín obtenido por $X$ e $Y$ todas son difeomorfas al plano.

\section{Acciones afines reales sobre una variedad compleja} A partir de aquí $\varphi:\left(\mathbb{R}_{>0} \times \mathbb{R}\right) \times M \rightarrow M$ es una acción diferenciable afín, donde $M$ es una variedad compleja y $\varphi_{(s, t)} \in \operatorname{Aut}(M) \quad$ (espacio de automorfismos holomorfos en $M$ ).

Sea

$$
\begin{aligned}
& z=\left(z_{1}, z_{2}, \cdots, z_{m}\right)=\left(x_{1}+i y_{1}, x_{2}+i y_{2}, \cdots, x_{m}+i y_{m}\right) \cong(x, y) \\
& \text { donde } \quad x=\left(x_{1}, x_{2}, \cdots, x_{m}\right) \quad \text { e }
\end{aligned}
$$

$y=\left(y_{1}, y_{2}, \cdots, y_{m}\right)$. En estas coordenadas locales se expresan los campos $X$ e $Y$ como sigue

$$
\begin{aligned}
& X=\sum_{j=1}^{m}\left(g_{j}^{1}(x, y) \frac{\partial}{\partial x_{j}}+h_{j}^{1}(x, y) \frac{\partial}{\partial y_{j}}\right), \\
& Y=\sum_{j=1}^{m}\left(g_{j}^{2}(x, y) \frac{\partial}{\partial x_{j}}+h_{j}^{2}(x, y) \frac{\partial}{\partial y_{j}}\right) .
\end{aligned}
$$

Sea $\quad J: T M \rightarrow T M$ el operador lineal de estructura casi-compleja inducido por la estructura compleja sobre $M$ (es decir $J\left(\frac{\partial}{\partial x_{j}}\right)=\frac{\partial}{\partial y_{j}} \quad$ y $\left.J\left(\frac{\partial}{\partial y_{j}}\right)=-\frac{\partial}{\partial x_{j}}\right)$. Entonces

$$
\begin{aligned}
& J X=\sum_{j=1}^{m}\left(-h_{j}^{1}(x, y) \frac{\partial}{\partial x_{j}}+g_{j}^{1}(x, y) \frac{\partial}{\partial y_{j}}\right) \\
& J Y=\sum_{j=1}^{m}\left(-h_{j}^{2}(x, y) \frac{\partial}{\partial x_{j}}+g_{j}^{2}(x, y) \frac{\partial}{\partial y_{j}}\right) .
\end{aligned}
$$

Por (2), se tiene $[X, J X]=[Y, J Y]=0$ y también $\tilde{X}=\frac{1}{2}(\mathrm{X}-i J \mathrm{X})$ e $\tilde{Y}=\frac{1}{2}(\mathrm{Y}-i J \mathrm{Y})$, luego se obtiene

$$
\tilde{X}=\sum_{j=1}^{m} f_{j}^{1}(z) \frac{\partial}{\partial z_{j}} \text { y } \quad \tilde{Y}=\sum_{j=1}^{m} f_{j}^{2}(z) \frac{\partial}{\partial z_{j}},
$$

donde

$$
f_{j}^{1}(z)=g_{j}^{1}(z)+i h_{j}^{1}(z) \mathrm{y}
$$




$$
f_{j}^{2}(z)=g_{j}^{2}(z)+i h_{j}^{2}(z)
$$

son holomorfos y $\frac{\partial}{\partial z_{j}}=\frac{1}{2}\left(\frac{\partial}{\partial x_{j}}-i \frac{\partial}{\partial y_{j}}\right)$ (véase $[4, \mathrm{pp}$. 126]). Es decir, los campos $\tilde{X}, \tilde{Y}$ son holomorfos. Como el flujo del campo $X$ es

$$
\begin{gathered}
X_{s}(x, y)=\varphi_{\left(e^{s}, 0\right)}(x, y)=\left(\varphi_{\left(e^{s}, 0\right)}^{1, x}(x, y), \cdots,\right. \\
\left.\varphi_{\left(e^{s}, 0\right)}^{m, x}(x, y), \varphi_{\left(e^{s}, 0\right)}^{1, y}(x, y), \cdots, \varphi_{\left(e^{s}, 0\right)}^{m, y}(x, y)\right) \\
X_{s}(x, y)=\varphi_{\left(e^{s}, 0\right)}(x, y)=\left(\varphi_{\left(e^{s}, 0\right)}^{1, x}(x, y), \cdots,\right. \\
\left.\varphi_{\left(e^{s}, 0\right)}^{m, x}(x, y), \varphi_{\left(e^{s}, 0\right)}^{1, y}(x, y), \cdots, \varphi_{\left(e^{s}, 0\right)}^{m, y}(x, y)\right)
\end{gathered}
$$

entonces el flujo de $J X$ está dado por

$$
\begin{gathered}
(J X)_{s}(x, y)=\left(-\varphi_{\left(e^{s}, 0\right)}^{1, y}(x, y), \cdots\right. \\
\left.-\varphi_{\left(e^{s}, 0\right)}^{m, y}(x, y), \varphi_{\left(e^{s}, 0\right)}^{1, x}(x, y), \cdots, \varphi_{\left(e^{s}, 0\right)}^{m, x}(x, y)\right)
\end{gathered}
$$

y para $\tilde{X}$ es

$$
\tilde{X}_{t+i s}(x, y)=X_{t}^{\circ}(J Y)_{s}(x, y)=(J Y)_{s}^{\circ} X_{t}(x, y) \text {. }
$$

Más adelante se presentan ejemplos donde $\tilde{X}_{t+i s}$ no es necesariamente un flujo periódico de período $2 \pi \mathrm{i}$, es decir no necesariamente se cumple

$\tilde{X}_{t+i s+2 \pi i}(z)=\tilde{X}_{t+i s}(z)$ para todo $z \in M, t+i s \in \mathbb{C}$.

Serán integrables los campos $\tilde{X}$ e $\tilde{Y}$ ?, es decir, existirá una foliación holomorfa singular de codimensión uno generado por estos campos. Para esto se requiere analizar la relación entre los campos con respecto al corchete de Lie.

Como [, ] es bilineal se cumple

$$
\begin{aligned}
{[\tilde{X}, \tilde{Y}] } & =\frac{1}{4}\{[X, Y]-i([X, J Y]+[J X, Y])-[J X, J Y]\} \\
& =\frac{1}{4}\{-Y-i([X, J Y]+[J X, Y])-[J X, J Y]\} \\
& =\frac{1}{4}\{-Y-i([X, J Y-J X+J X]+[J X-J Y+J Y, Y])-[J X, J Y]\} \\
& =\frac{1}{4}\{-Y-i([X, J Y-J X]+[J X-J Y, Y])-[J X, J Y]\} \\
& =\frac{1}{4}\{-Y-i([J(X-Y), X+Y])-[J X, J Y]\}
\end{aligned}
$$

por otro lado, por el teorema de Newlander-Nirenberg (véase [8, pp. 96] y [9])

$$
[J X, J Y]-J[J X, Y]-J[X, J Y]-[X, Y]=0,
$$

entonces $\quad Y+[J X, J Y]=J[J(X-Y), X+Y]$ reemplazando en la ecuación anterior tendremos

$$
[\tilde{X}, \tilde{Y}]=-\frac{1}{2} i \tilde{Z}
$$

donde $Z=[J(X-Y), X+Y]$.

Haciendo $Z=\sum_{i=1}^{n}\left(u_{i} \frac{\partial}{\partial x_{i}}+v_{i} \frac{\partial}{\partial y_{i}}\right)$ tendremos que

$$
\begin{aligned}
u_{i}= & \sum_{j=1}^{m}\left\{\left(h_{j}^{2}-h_{j}^{1}\right) \frac{\partial}{\partial x_{j}}\left(g_{i}^{1}+g_{i}^{2}\right)+\left(g_{j}^{1}-g_{j}^{2}\right) \frac{\partial}{\partial y_{j}}\left(g_{i}^{1}+g_{i}^{2}\right)-\right. \\
& \left.\left(g_{j}^{1}+g_{j}^{2}\right) \frac{\partial}{\partial x_{j}}\left(h_{i}^{2}-h_{i}^{1}\right)-\left(h_{j}^{1}+h_{j}^{2}\right) \frac{\partial}{\partial y_{j}}\left(h_{i}^{2}-h_{i}^{1}\right)\right\} \\
v_{i}= & \sum_{j=1}^{m}\left\{\left(h_{j}^{2}-h_{j}^{1}\right) \frac{\partial}{\partial x_{j}}\left(h_{i}^{1}+h_{i}^{2}\right)+\left(g_{j}^{1}-g_{j}^{2}\right) \frac{\partial}{\partial y_{j}}\left(h_{i}^{1}+h_{i}^{2}\right)-\right. \\
& \left.\left(g_{j}^{1}+g_{j}^{2}\right) \frac{\partial}{\partial x_{j}}\left(g_{i}^{1}-g_{i}^{2}\right)-\left(h_{j}^{1}+h_{j}^{2}\right) \frac{\partial}{\partial y_{j}}\left(g_{i}^{1}-g_{i}^{2}\right)\right\} .
\end{aligned}
$$

Proposición 4 Sean $\tilde{X}, \tilde{Y}$ y $Z$ los campos vectoriales definidos anteriormente. Las siguientes afirmaciones son equivalentes:
a) $[\tilde{X}, \tilde{Y}]=-\tilde{Y}$.
b) $Z=-2 J Y$.
c) $[J X, J Y]=Y$.

Demostración: (a) $\diamond$ (b): La condición $[\tilde{X}, \tilde{Y}]=$ $-\tilde{Y}$. implica que $-\frac{1}{2} \widetilde{Z}=-\tilde{Y}$. Ahora usando la definición de $\tilde{Y}$ y $\tilde{Z}$ tendremos

$$
\frac{1}{2} J Z+i \frac{1}{2} Z=Y-i J Y .
$$

De esto se obtiene $Z=-2 J Y$.

(b) $\diamond$ (c): Por el teorema de Newlander-Nirenberg se tiene

$$
[J X, J Y]-J[J X, Y]-J[X, J Y]-[X, Y]=0,
$$

aplicando el operador $J$ se tiene:

$J[J X, J Y]-J^{2}[J X, Y]-J^{2}[X, J Y]-J[X, Y]=0$.

Por la definición del operador $J$, se obtiene

$$
J[J X, J Y]+[J X, Y]+[X, J Y]+J Y=0
$$

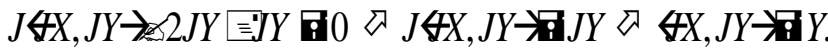

(c) $\gtrsim$ (a): Usando la condición $[J X, J Y]=Y$

junto con el teorema de Newlander-Nirenberg

$$
Z=[J(X-Y), X+Y]=[J X, Y]-[J Y, X]
$$

$$
=-J[J X, J Y]-J Y=-J Y-J Y=-2 J Y .
$$

Luego

$$
\begin{aligned}
{[\tilde{X}, \tilde{Y}] } & =-\frac{1}{2} i \tilde{Z}=-\frac{1}{2} i\left(\frac{Z-i J Z}{2}\right)=-\frac{1}{2} i\left(\frac{-2 J Y-2 i Y}{2}\right) \\
& =-\frac{1}{2} i\left(\frac{-2 J Y-2 i Y}{2}\right)=-\tilde{Y} .
\end{aligned}
$$


Proposición 5 Sean $\tilde{X}, \tilde{Y}$ y $Z$ los campos vectoriales definidos anteriormente. Las siguientes afirmaciones son equivalentes:
a) $[\tilde{X}, \tilde{Y}]=0$.
b) $Z=0$.
c) $[J X, J Y]=-Y$.

Demostración: Similar a la prueba de la proposición anterior.

Ya que $\tilde{X}$ no necesariamente es periódico entonces tendremos un mapeo multivaluado $\tilde{\varphi}=$ $\mathrm{C}^{*} \times \mathrm{C}^{m} \times M \rightarrow M, \tilde{\varphi}_{(s, t)}=\tilde{Y}_{t}^{\circ} \tilde{X}_{l n s}$.

El conjunto $\mathrm{C}^{*} \times \mathrm{C}$ es un grupo de Lie complejo con la operación $\circ$, definido por:

$$
\begin{aligned}
& \text { Luego } \\
& \begin{aligned}
\tilde{X}= & \frac{1}{2}\left[\left(x_{1}+i y_{1}\right) \frac{\partial}{\partial x_{1}}+\left(y_{1}-i x_{1}\right) \frac{\partial}{\partial y_{1}}+\lambda_{2}\left(x_{2}+i y_{2}\right) \frac{\partial}{\partial x_{2}}+\lambda_{2}\left(y_{2}-i x_{2}\right) \frac{\partial}{\partial y_{2}}+\right. \\
& \left.\lambda_{3}\left(x_{3}+i y_{3}\right) \frac{\partial}{\partial x_{3}}+\lambda_{3}\left(y_{3}-i x_{3}\right) \frac{\partial}{\partial y_{3}}\right] \\
= & \frac{1}{2}\left[\left(x_{1}+i y_{1}\right) \frac{\partial}{\partial x_{1}}-i\left(x_{1}+i y_{1}\right) \frac{\partial}{\partial y_{1}}+\lambda_{2}\left(x_{2}+i y_{2}\right) \frac{\partial}{\partial x_{2}}-\lambda_{2} i\left(x_{2}+i y_{2}\right) \frac{\partial}{\partial y_{2}}+\right. \\
& \left.\lambda_{3}\left(x_{3}+i y_{3}\right) \frac{\partial}{\partial x_{3}}-\lambda_{3}\left(x_{3}+i y_{3}\right) \frac{\partial}{\partial y_{3}}\right] \\
= & \frac{1}{2}\left[z_{1} \frac{\partial}{\partial x_{1}}-i z_{1} \frac{\partial}{\partial y_{1}}+\lambda_{2} z_{2} \frac{\partial}{\partial x_{2}}-\lambda_{2} i z_{2} \frac{\partial}{\partial y_{2}}+\lambda_{3} z_{3} \frac{\partial}{\partial x_{3}}-\lambda_{3} i z_{3} \frac{\partial}{\partial y_{3}}\right] \\
= & \frac{1}{2}\left[z_{1}\left(\frac{\partial}{\partial x_{1}}-i \frac{\partial}{\partial y_{1}}\right)+\lambda_{2} z_{2}\left(\frac{\partial}{\partial x_{2}}-i \frac{\partial}{\partial y_{2}}\right)+\lambda_{3} z_{3}\left(\frac{\partial}{\partial x_{3}}-i \frac{\partial}{\partial y_{3}}\right)\right] \\
= & z_{1} \frac{\partial}{\partial z_{1}}+\lambda_{2} z_{2} \frac{\partial}{\partial z_{2}}+\lambda_{3} z_{3} \frac{\partial}{\partial z_{3}}
\end{aligned}
\end{aligned}
$$

Definición 12 Una acción holomorfa $\varphi:\left(\mathbb{C}^{*} \times \mathbb{C}\right) \times$ $M \rightarrow M$ del grupo $\left(\mathbb{C}^{*} \times \mathbb{C}, \circ\right)$ sobre una variedad compleja $M$ de dimensión por lo menos tres es llamado acción holomorfa afín.

Teorema 3 Si $M=\mathbb{C} \quad$ y $[\mathrm{JX}, J Y]=Y$ con $\tilde{X}$ cuyo flujo es $2 \pi i$ periódico entonces $\tilde{\varphi}$ es una acción holomorfa bien definida del grupo de Lie $\left(\mathrm{C}^{*} \times \mathrm{C}, \circ\right)$ sobre $\mathrm{C}^{m}$.

Demostración: Cuando la variedad $M$ es $\mathrm{C}^{m}$ por el Corolario 2.2 de [4, pp. 130] los campos $\tilde{X}$ e $\tilde{Y}$ son $\mathrm{C}_{\text {-completos. }}$

Por hipótesis $[J X, J Y]=Y$ y por la Proposición 5 se obtiene $[\tilde{X}, \tilde{Y}]=-\tilde{Y}$. Por otro lado como el campo $\tilde{X}$ tiene flujo $2 \pi$ i periódico, $\tilde{\varphi}$ está bien definido.

Usando la Proposición 2 de [5, pp. 22] $\tilde{\varphi}$ es una acción holomorfa del grupo $\left(\mathrm{C}^{*} \times \mathrm{C}, \circ\right)$ sobre $\mathrm{C}^{m}$.

Demostración: Similar a la prueba de la proposición anterior.

Ejemplo 2 Dados los campos

$$
\begin{gathered}
X=\left(x_{1} \frac{\partial}{\partial x_{1}}+y_{1} \frac{\partial}{\partial y_{1}}\right)+\left(\lambda_{2} x_{2} \frac{\partial}{\partial x_{2}}+\lambda_{2} y_{2} \frac{\partial}{\partial y_{2}}\right)+ \\
\left(\lambda_{3} x_{3} \frac{\partial}{\partial x_{3}}+\lambda_{3} y_{3} \frac{\partial}{\partial y_{3}}\right)
\end{gathered}
$$

donde $\lambda_{2}, \lambda_{3} \in Z$ e $Y=\frac{\partial}{\partial x_{1}}$. Estos campos satisfacen la $[X, Y]=-Y$.

Se tiene

$$
\begin{aligned}
& J X=\left(x_{1} \frac{\partial}{\partial y_{1}}-y_{1} \frac{\partial}{\partial x_{1}}\right)+\left(\lambda_{2} x_{2} \frac{\partial}{\partial y_{2}}-\lambda_{2} y_{2} \frac{\partial}{\partial x_{2}}\right)+ \\
& \left(\lambda_{3} x_{3} \frac{\partial}{\partial y_{3}}-\lambda_{3} y_{3} \frac{\partial}{\partial x_{3}}\right)
\end{aligned}
$$

$\tilde{X}$ está dado por $\tilde{X}_{t}(z)=\left(e^{t} z_{1}, e^{\lambda_{2} t} z_{2}, e^{\lambda_{3} t} z_{3}\right)$. Como $\lambda_{2}, \lambda_{3} \in Z, \tilde{X}_{t}$ es periódica de periodo $2 \pi \mathrm{i}$.

En este ejemplo se observa que los campos $X$ y $J X$ no tienen flujo periódico pero sin embargo el campo holomorfo $\widetilde{X}$ tiene flujo periódico de periodo $2 \pi \mathrm{i}$.

Ejemplo 3 Sea $\varphi:\left(\mathbb{R}_{>0} \times \mathbb{R}\right) \times \mathbb{C}^{3} \rightarrow \mathbb{C}^{3}$ dado por

$$
\begin{gathered}
\varphi_{(s, t)}(x, y)=\left(\left(-k_{1} s^{-1}+x_{2} \ln s+x_{1}+k_{1}\right) s+\right. \\
\left(-k t,\left(-k_{2} s^{-1}+y_{2} \ln s+y_{1}+k_{2}\right) s,\right. \\
\left(s^{\lambda_{2}} x_{2}, s^{\lambda_{2}} y_{2}, s^{\lambda_{3}} x_{3}, s^{\lambda_{3}} y_{3}\right), \\
\text { para todo }(s, t) \in \mathrm{R}_{>0} \times \mathrm{R} .
\end{gathered}
$$

Es una acción diferenciable afín. Calculando los campos asociados a $\varphi$.

$$
\begin{aligned}
X(x, y)= & \left.\frac{\partial}{\partial s} \varphi_{\left(e^{s}, 0\right)}(x, y)\right|_{s=0} \\
= & \frac{\partial}{\partial s}\left(\left(-k_{1} e^{-s}+x_{2} s+x_{1}+k_{1}\right) e^{s},\left(-k_{2} e^{-s}+y_{2} s+y_{1}+k_{2}\right) e^{s},\right. \\
& \left.e^{\lambda_{2} s} x_{2}, e^{\lambda_{2} s} y_{2}, e^{\lambda_{3} s} x_{3}, e^{\lambda_{3} s} y_{3}\right)\left.\right|_{s=0}
\end{aligned}
$$$$
=\left(k_{1}+x_{1}+x_{2}, k_{2}+y_{1}+y_{2}, \lambda_{2} x_{2}, \lambda_{2} y_{2}, \lambda_{3} x_{3}, \lambda_{3} y_{3}\right)
$$$$
=\left(k_{1}+x_{1}+x_{2}\right) \frac{\partial}{\partial x_{1}}+\left(k_{2}+y_{1}+y_{2}\right) \frac{\partial}{\partial y_{1}}+\lambda_{2} x_{2} \frac{\partial}{\partial x_{2}}+\lambda_{2} y_{2} \frac{\partial}{\partial y_{2}}
$$$$
+\lambda_{3} x_{3} \frac{\partial}{\partial x_{3}}+\lambda_{3} y_{3} \frac{\partial}{\partial y_{3}}
$$ 


$$
\begin{aligned}
Y(x, y) & =\left.\frac{\partial}{\partial t} \varphi_{(1, t)}(x, y)\right|_{t=0} \\
& =(1,0,0,0,0,0) \\
& =\frac{\partial}{\partial x_{1}} .
\end{aligned}
$$

Estos campos satisfacen $[X, Y]=-Y$.

Los campos $\tilde{X}$ e $\tilde{Y}$ están dados por

$$
\begin{aligned}
& \tilde{X}=\left(k+z_{1}+z_{2}\right) \frac{\partial}{\partial z_{1}}+\lambda_{2} z_{2} \frac{\partial}{\partial z_{2}}+\lambda_{3} z_{3} \frac{\partial}{\partial z_{3}} \\
& \tilde{Y}=\frac{\partial}{\partial z_{1}}
\end{aligned}
$$

donde $k=k_{1}+k_{2} i \quad$ y $\quad z_{j}=x_{j}+y_{j} i$, para todo $j=1,2,3$.

También se verifica $[\tilde{X}, \tilde{Y}]=-\tilde{Y}$, y el flujo del campo $\tilde{X}$ esta dado por

$$
\tilde{X}_{t}(z)=\left(\left(-k e^{-t}+z_{1}+z_{2} t+k\right) e^{t}, e^{\lambda_{2} t} z_{2}, e^{\lambda_{3} t} z_{3}\right)
$$

\section{para todo $t \in \mathrm{C}$}

el cual no es periódica, dado que $\tilde{X}_{t+2 \pi i}(z) \neq \tilde{X}_{t}(z)$.

\section{Resultados y discusión}

Sea $\varphi: A f f(\mathrm{R})^{+} \times M \rightarrow M \quad$ una acción diferenciable afín de $\operatorname{Aff}(\mathrm{R})^{+}$sobre una variedad diferenciable $M$, entonces existen dos campos diferenciables $\mathbb{R}$-completos $X$ e $Y$ tales que: $[X, Y]=-Y$. Si $X_{s}$ e $Y_{t}$ denotan los flujos de los campos $X$ e $Y$ entonces $\varphi_{(s, t)}=Y_{t} \circ X_{\ln s}$. Lo recíproco también es cierto, es decir si tenemos dos campos diferenciables $\mathrm{R}$-completos $X$ e $Y$ tal que $\quad[X, Y]=-Y \quad, \quad$ entonces $\varphi:\left(\mathrm{R}_{>0} \times \mathrm{R}\right) \times M \rightarrow M \quad$ dado por $\varphi_{(s, t)}=Y_{t} \circ X_{\ln s}$ es una acción diferencial afín.

Sea $\varphi: A f f(\mathrm{R})^{+} \times M \rightarrow M \quad$ una acción diferenciable afín de $A f f(\mathrm{R})^{+}$sobre una variedad compleja $M$ tal que $\varphi_{(s, t)} \in \operatorname{Aut}(M)$, para todo $(s, t) \in A f f(\mathrm{R})^{+}$. Sea $J: T M \rightarrow T M \quad$ el operador de estructura casi-compleja inducida por la estructura compleja sobre $M$. Definimos $\tilde{X}=$ $\frac{1}{2}(\mathrm{X}-i J \mathrm{X}) \quad$ e $\quad \tilde{Y}=\frac{1}{2}(\mathrm{Y}-i J \mathrm{Y}) \quad$. Como

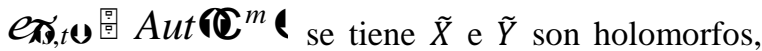
$[X, J X]=0$ y $[Y, J Y]=0$.
Usando las propiedades del corchete de Lie y el Teorema Newlander-Niremberg se prueba las siguientes relaciones equivalentes:

$[\tilde{X}, \tilde{Y}]=-\tilde{Y} \Leftrightarrow Z=-2 J Y \Leftrightarrow[J X, J Y]=Y$, donde $Z=[J(X-Y), X+Y]$.

Sea $\varphi: A f f(\mathrm{R})^{+} \times \mathrm{C}^{m} \rightarrow \mathrm{C}^{m}$ una acción diferenciable afín de $\operatorname{Aff}(\mathrm{R})^{+}$sobre una variedad compleja $\mathrm{C}^{m}$ tal que $\varphi_{(s, t)} \in \operatorname{Aut}\left(\mathrm{C}^{m}\right)$, para todo $(s, t) \in A f f(\mathrm{R})^{+}$. Cuando la variedad es $\mathrm{C}^{m}$ los campos $\tilde{X}$ e $\tilde{Y}$ son $\mathrm{C}$-completos. Asumiendo que se cumple una de las identidades equivalentes y si $\tilde{X}$ es un campo cuyo flujo es necesariamente $2 \pi$ i periódico entonces se llega a la caracterización de acción holomorfa afín usando un resultado sobre acciones holomorfas y con esto se obtiene el siguiente resultado.

\section{Conclusiones}

Sea $\varphi: \operatorname{Aff}(\mathrm{R})^{+} \times \mathrm{C}^{m} \rightarrow \mathrm{C}^{m}$ una acción diferenciable afín y $\varphi_{(s, t)} \in \operatorname{Aut}\left(\mathrm{C}^{m}\right)$, para todo $(s, t) \in \operatorname{Aff}(\mathrm{R})^{+}$. Sea $X \quad$ e $Y$ campos $\mathbb{R}$-completos asociado a $\varphi . \mathrm{Si}[J X, J Y]=Y$ con $\tilde{X}$ cuyo flujo es $2 \pi \mathrm{i}$ periódico, entonces $\tilde{\varphi}=$ $\left(\mathbb{C}^{*} \times \mathbb{C}, \circ\right) \times \mathbb{C}^{m} \rightarrow \mathbb{C}^{m}$ definido por $\tilde{\varphi}_{(s, t)}=$ $\tilde{Y}_{t}^{\circ} \tilde{X}_{\text {lns }}$ es una acción holomorfa afín.

\section{Literatura citada}

[1] C. Camacho y A. Lins Neto: Teoría geométrica das folheações. IMPA, (1979).

[2] R. Coutinho: Linearization of affine group actions; Rio de Janeiro (2008).

[3] Dmitri N. Akhiezer: Lie Group Actions in Complex Analysis. Aspects of Mathematics, Moscow, (1994).

[4] F. Forsteric: Actions of $\mathbb{R}, \exists$ and $\mathbb{C}, \exists$ on complex manifolds. Department of Mathematics, University of Wisconsin, Madison, año 1996, pp.123-153.

[5] F. Forstnerič: Stein manifolds holomorphic mappings. Springer-Verlang Berlin Heidelberg, Vol.56 (2011).

[6] J. F. Plante: Locally free affine group actions. Transactions of American Mathematical Society, Vol. 259, número 2, Julio de 1980, pp. 499-456.

[7] J. M. Sotomayor Tello: Lições de equações diferenciais analititcas. Instituto de Matemática Pura y Aplicada (Proyecto Euclides), (1979).

[8] C. Camacho y P. Sad: Pontos singulares de equações diferenciais analiticas. $16^{\circ}$ Coloquio Brasileiro de Matemática, IMPA (1987).

[9] A. Newlander y L. Nirenberg: Complex coordinates in almost complex manifolds. Ann. of Math. 65 (1957), pp. 391-428. 